\title{
GC-MS: herramienta fundamental para el análisis de drogas de uso ilícito
}

\author{
Elena Stashenko*, Jairo René Martínez \\ Centro de Investigación de Excelencia - CENIVAM, Centro de Cromatografía y Espectrometría de Masas - CROM-MASS, \\ Escuela de Química, Universidad Industrial de Santander - UIS, Bucaramanga, Colombia \\ e-mail:elena@tucan.uis.edu.co
}

\section{Resumo}

La determinación cromatográfica de drogas de uso ilícito presenta retos importantes en varias etapas de la cadena analítica debido a la volatilidad relativamente baja de los analitos, a la diversidad grande de los posibles contaminantes y a las concentraciones bajas en las que se encuentran algunas sustancias de interés. La preparación de la muestra, el uso de agentes de derivación, los modos de operación del espectrómetro de masas para la detección y los diversos campos de aplicación del análisis GC-MS de sustancias controladas se discuten en este artículo junto con la presentación de algunos ejemplos recientes de estas aplicaciones.

Palavras-chave

Cromatografía de gases; cromatografía de gases acoplada a espectrometría de masas; sustancias controladas; drogas.

\section{GC-MS: fundamental tool for the analysis of drugs of illicit use}

\section{Abstract}

The chromatographic determination of illicit drugs poses important challenges at various stages along the analytical procedure, due to the low volatility of the analytes, the diversity of potential contaminants, and to the low concentrations of certain substances of interest. Sample preparation, the use of derivatization agents, different operating modes of the mass spectrometer for the detection, and the diverse application fields of the GC-MS analysis of illicit drugs are discussed in this article together with the presentation of some examples of these applications.

Keywords

Gas chromatography; gas chromatography-mass spectrometry; controlled substances; drugs.

\section{Introducción}

Las drogas de uso ilícito, consumidas con el propósito final de alterar el estado de conciencia o percepción, forman parte de las llamadas sustancias controladas, cuyo análisis confiable es muy importante en diferentes ramas de la ciencia, por ejemplo, en medicina, toxicología, control de doping, asuntos legales (ciencias forenses), pruebas para empleo o supervisión laboral, accidentes 
de tránsito, programas de rehabilitación, entre otras áreas. Existen diferentes escenarios posibles, cuando se necesita determinar la presencia de sustancias ilícitas (drogas) y cuantificarlas. Se han desarrollado diferentes protocolos de análisis (muestreo, extracción, determinación cualitativa y cuantitativa) de estas sustancias, que se encuentran tanto en forma (relativamente) pura (e.g., clorhidrato de cocaína), como en mezclas complejas (street drugs, drogas de la calle o de diseño) o a nivel de trazas (superficies de contacto, fluidos fisiológicos). La detección de drogas en toxicología forense puede realizarse tanto en muestras post-mortem (en cadáveres), como pre-mortem. Las muestras post-mortem son los especímenes recolectados durante la autopsia; entre éstos, pueden figurar -según su estado $y$ la disponibilidad-, sangre, humor vítreo, orina, bilis, hígado y algunos otros (contenido estomacal, cabello, uñas, etc.). Las muestras pre-mortem, obtenidas de individuos vivos, generalmente son orina, sangre o algunos especímenes más "exóticos", menos comunes, por ejemplo, saliva, sudor, cabello, uñas y hasta lágrimas. Por supuesto, la muestra más típica es la orina, es un desecho metabólico del organismo, su obtención no es invasiva, y el peligro de contaminación microbiana para un analista es menor que cuando se trabaja con la sangre; además la orina es un líquido, con pocas interferencias presentes (proteínas, grasa) y con relativamente alta concentración de la droga o de sus metabolitos.

El método de análisis de sustancias controladas, de mayor uso a lo largo de la historia, ha sido la cromatografía, de capa fina, líquida y de gases. El método analítico, que reviste alta selectividad y sensibilidad y que permite confirmar la naturaleza química de la droga ha sido la cromatografía de gases (GC) acoplada a espectrometría (MS). Todas las muestras toxicológicas requieren, para su subsiguiente análisis por GC-MS, una preparación previa, i.e., extracción, limpieza, a menudo, derivación de analitos, para potencializar la respuesta del detector, aumentar la volatilidad y la termoestabilidad del analito o mejorar la forma de su señal cromatográfica (pico).

En general, los analitos (sustancias controladas, sus derivados o metabolitos) se podrían dividir en dos grupos grandes: (1) muestras bulk (en volumen), por ejemplo, la droga incautada, pastillas distribuidas al por menor (street drug) en la calle, productos fabricados en un laboratorio clandestino, entre otros y (2) a nivel de trazas; entre estos últimos figuran muestras toxicológicas (fluidos, órganos, tejidos), muestras recolectadas de las superficies de contacto con la droga, manos, empaques, tapetes, ropa, etc. Un grupo de analitos muy sui generis lo representan las sustancias encontradas en los laboratorios clandestinos, donde se extrae (e.g., cocaína, morfina) o se sintetiza la droga de uso ilícito (e.g., anfetaminas). En estos laboratorios clandestinos, los materiales de partida, los disolventes o los productos de síntesis obtenidos pueden tener rótulos o etiquetas en los recipientes muy distintos a su verdadero contenido y hay que tener mucha precaución para llevar a cabo la marcha analítica y la determinación de la naturaleza química de las sustancias.

Los análisis de sustancias controladas pueden dividirse en dos grandes grupos, según el propósito o alcance final: (1) Pruebas presuntivas (preliminares o de tamizaje, screening) y (2) Pruebas confirmatorias. Las pruebas presuntivas comprenden las reacciones de color, que se realizan in situ con un kit de reactivos específicos (por ejemplo, reactivo de Ehrlich, prueba de Marquis, reactivo de Mandelin, prueba de Scott, entre otros) que se usan, por ejemplo, en aeropuertos, puertos marítimos, aduanas, etc.; la cromatografía de capa fina (TLC, Thin-Layer Chromatography, por sus siglas en inglés), sobre todo, la TLC de alta eficiencia, que se practica en 
muchos laboratorios toxicológicos, por ser un método rápido, relativamente sencillo, para establecer la presencia de una droga, e.g., en casos de intoxicaciones, sobredosis, etc.; los inmunoensayos (por ejemplo, RIA, Radioimmunoassay; ELISA, Enzyme-Linked Immunosorbent Assay; Enzyme-multiplied immunoassay technique, EMIT, por mencionar algunos), que permiten seleccionar - de un gran número de muestras-, aquellas con la respuesta positiva, para seguir luego con su análisis confirmatorio. La cromatografía de gases tradicionalmente es el método más empleado para análisis de drogas de control y sus metabolitos; típicamente se usan detectores convencionales, e.g., FID (detector de ionización en llama), NPD (detector de nitrógeno y fósforo) o ECD (detector de captura de electrones, para derivados), se comparan los tiempos de retención $\left(\mathrm{t}_{\mathrm{R}}\right)$ de las sustancias (drogas) con los del material de referencia (patrones); la coincidencia de los $t_{R}$ de los analitos (drogas $y$ sustancias-patrón) resulta ser un evento necesario, pero no suficiente para la identificación confirmatoria de la sustancia. Para la confirmación positiva es menester no solamente tener el material de referencia, sino disponer de un sistema de detección espectroscópica. Entre los más usados, un detector selectivo de masas (MSD), que permite obtener y comparar los espectros de masas de la sustancia "X" y del compuesto-patrón. Las columnas cromatográficas capilares más usadas son de 5\%-fenil-poli(metilsiloxano), de 25-30 m de longitud y $0,25 \mathrm{~mm}$ de diámetro interno (e.g., DB-5, HP-5, ultra 2, entre otras marcas comerciales). Para compuestos presentes a nivel de trazas (e.g., fluidos fisiológicos), el modo de inyección al GC es splitless o pulsed splitless; para analitos termoinestables, cold on-column; mientras que las muestras bulk (en volumen), junto con otras técnicas de análisis físico-químico y espectroscópico (NMR, FT-IR, difracción de rayos $\mathrm{X}$, etc.) pueden ser analizadas, inyectándolas al GC en modo split. Para una mejor separación de los componentes y su cuantificación, las mezclas complejas de algunas drogas de la calle (street drugs, club drugs), que contienen principio(s) activo(s), aditivos y adulterantes, solventes residuales, productos de descomposición o reactivos de partida, se analizan en columnas más largas (50-60 m) o empleando la cromatografía multidimensional. En la Figura 1 aparece el cromatograma típico del extracto de una pastilla decomisada (club drug), analizada por GC-MS (ionización electrónica, EI, $70 \mathrm{eV}$ ).

La clasificación de las drogas de uso ilícito se basa fundamentalmente en los efectos fisiológicos que éstas producen en el organismo y no según su estructura química. Los grupos principales de sustancias controladas en ciencias forenses comprenden: 1. Estimulantes del sistema nervioso central (e.g., cocaína, anfetaminas); 2. Depresores del sistema nervioso central (entre muchos otros, alcohol, opio, opioides naturales, semi-sintéticos y sintéticos, benzodiacepinas); 3. Alucinógenos (LSD, mescalina, psilocibina y psilocina, algunos alcaloides tropánicos, triptaminas y sus derivados, muchos otros compuestos sintéticos, e.g., fenciclidina, PCP, entre otros); 4. Canabinoides (productos derivados de la marihuana, material vegetal, resina, hachís, aceite de hachís); 5. Antidepresivos de primera, segunda y tercera generaciones y 6 . Inhalantes (Hidrocarburos alifáticos, aromáticos, halogenados, fluorocarbonados, acetona, óxido nitroso, anestésicos gaseosos, entre otros).

La preparación de especímenes para determinar la presencia de sustancias controladas, sobre todo, cuando estos compuestos o sus metabolitos se encuentran a nivel de trazas, implica la realización de toda la cadena de preparación de la muestra, i.e., muestreo, extracción y limpieza, derivación, análisis presuntivo y confirmatorio 


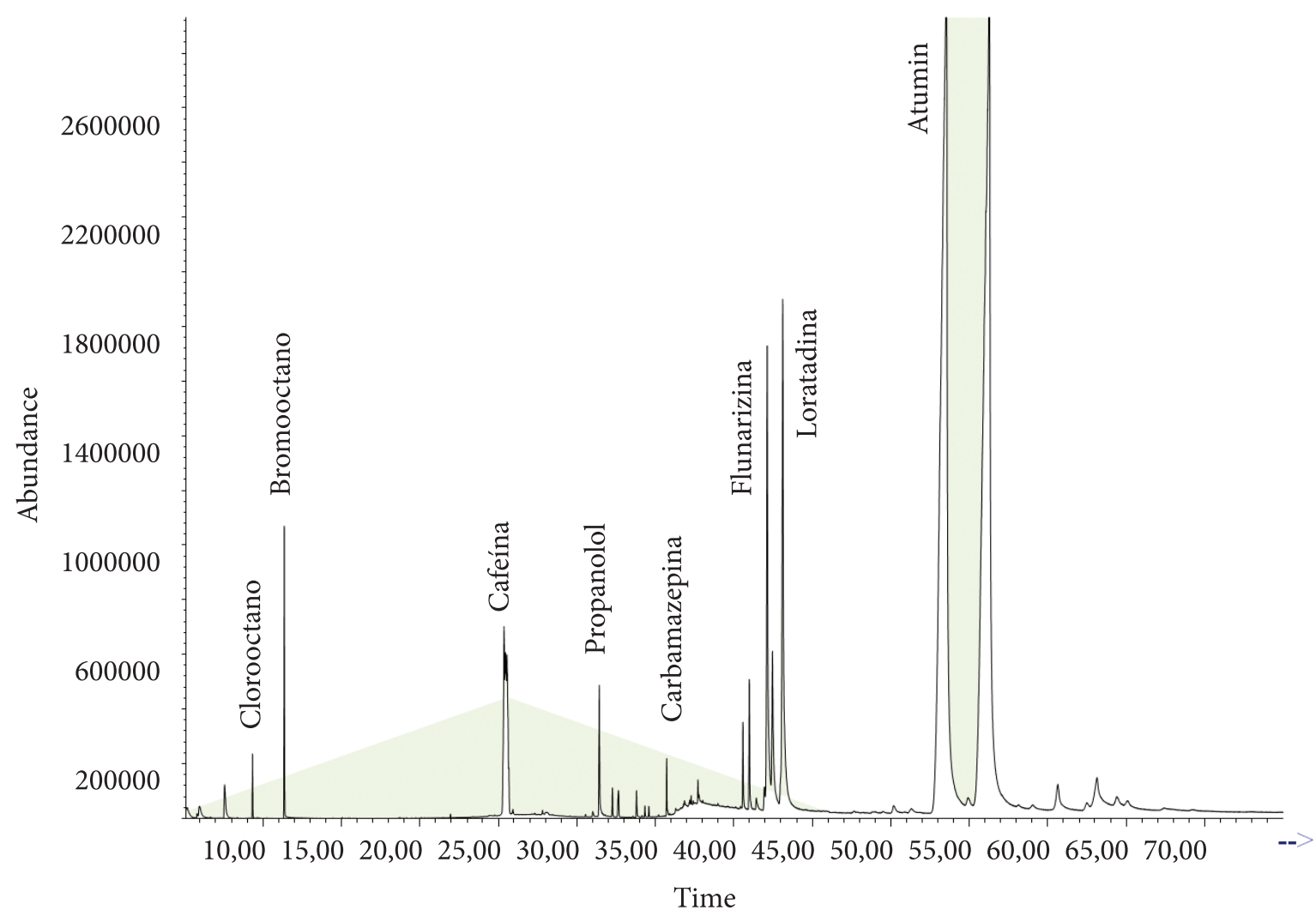

Figura 1 Perfil cromatográfico típico (TIC) del extracto de una pastilla de droga de uso ilícito (club drug). GC-MS. $\mathrm{El}, 70 \mathrm{eV}$. Columna DB-5MS, $60 \mathrm{~m}$.

de analitos, interpretación de datos, junto con el análisis de los respectivos blancos, negativo y positivo, y obtención de las figuras analíticas de mérito. Los métodos de extracción más usados son extracción líquido-líquido (LLE), en fase sólida (SPE), micro-extracción en fase sólida (SPME). Las operaciones-base están relacionadas también con la precipitación de proteínas, centrifugación, filtración, adición de sal (salting out) y el cambio de $\mathrm{pH}$, según el $\mathrm{pKa}$ de la sustancia; la determinación de los disolventes ocultos y la perfilación (profiling) de drogas, que comprende la identificación de impurezas, productos de descomposición, trazas de reactivos de partida, adulterantes, artefactos, etc., se hace a menudo por métodos headspace, en combinación con otras técnicas de extracción, e.g., SPME, con el uso de un disolvente o un adsorbente. La sensibilidad y la selectividad en el análisis se logran obteniendo fragmentogramas de masas, registrados en modo de monitoreo del ion o iones seleccionados (SIM). En la Figura 2 aparecen corrientes iónicas obtenidas en modo full scan (barrido completo) y SIM (iones seleccionados en $m / z 94,138,303$ ) de un extracto aislado con fluido supercrítico (SFE, $\mathrm{CO}_{2}$ ) de frutos de Datura sp. (Familia Solanácea), que contiene la escopolamina, utilizada ilícitamente por los delincuentes para desorientar y paralizar la fuerza de voluntad y autocontrol de la víctima. El uso de sistemas de detección de masas tándem, por ejemplo, el de triple cuadrupolo (GC/MS-QqQ), permite elevar sobremanera la especificidad de la detección. En la Figura 3 aparecen corrientes iónicas de un extracto de una bebida (infusión) a base de diferentes plantas, que contiene trazas de cocaína, obtenidas en los modos: (A) full scan; (B) SIM de iones diagnóstico de la cocaína en 


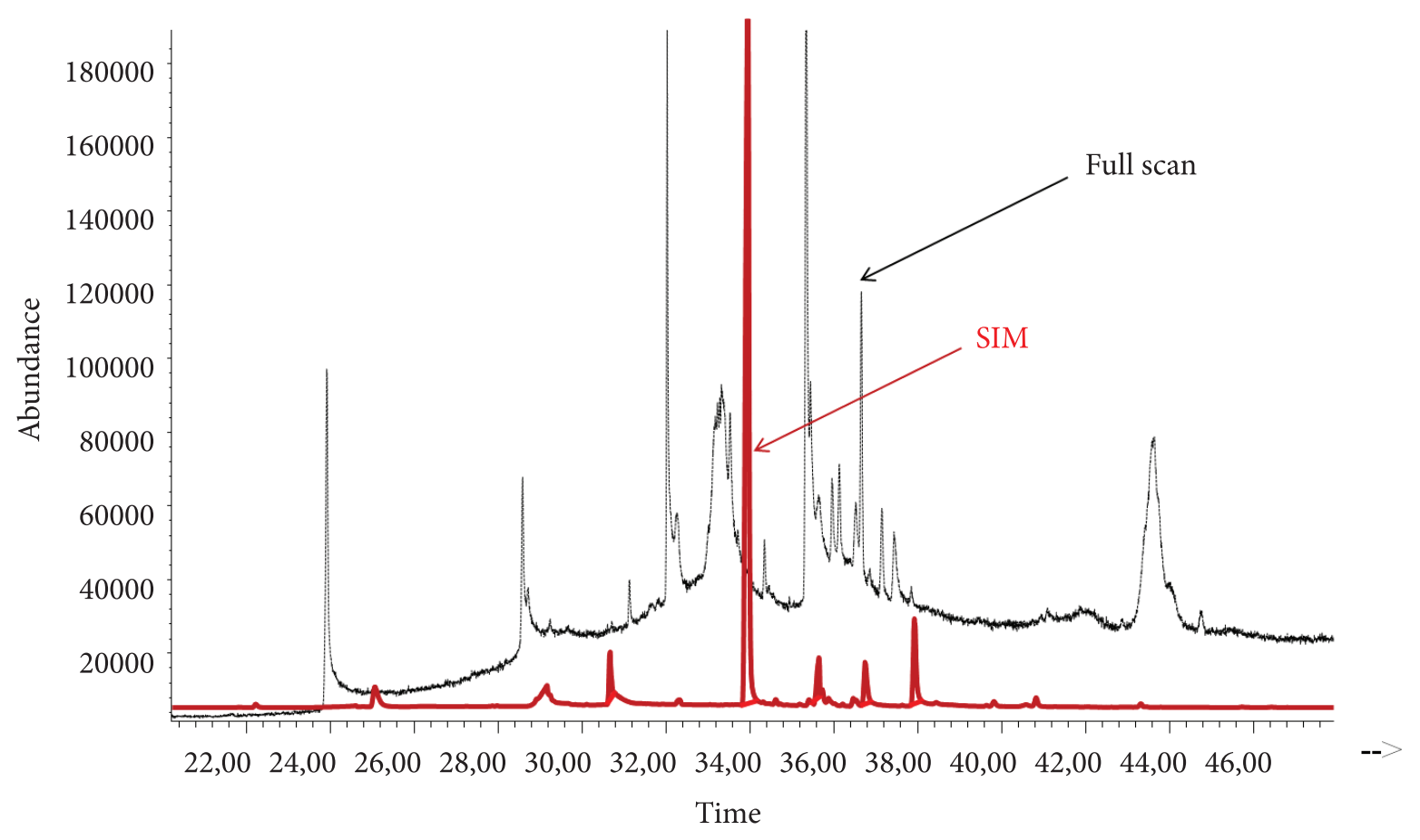

Figura 2 Corriente iónica total (TIC) de un extracto aislado con fluido supercrítico (SFE, $\mathrm{CO}_{2}$ ) de frutos de Datura sp. (Familia Solanácea) y fragmentograma de masas del mismo extracto, obtenido con base en iones diagnóstico de la escopolamina, i.e., m/z 94, 138 y 303.

$m / z 82,182$ y 303 y $(\mathbf{C})$ registrando la transición iónica $303 \rightarrow 182$, que permite aumentar mucho la especificidad del análisis. En las siguientes secciones se presentan ejemplos típicos del análisis GC-MS de drogas ilícitas.

\section{Análisis GC-MS unidimensional}

Existen varios propósitos que determinan el enfoque a seguir en el análisis cromatográfico de muestras que contengan drogas de uso ilícito. Se ha normalizado una gran cantidad de procedimientos de cuantificación de analitos particulares, tales como cocaína, opioides, anfetaminas, benzodiacepinas y drogas de diseño. En estos procedimientos normalmente se utiliza la derivación química y la determinación instrumental se realiza con el modo SIM de operación del espectrómetro de masas. El foco de atención en estos casos es la sustancia psicoactiva y su presencia se determina en matrices tan diversas como cabello, fluido oral, orina, sangre y placenta. En muchas otras situaciones el interés forense se centra en los otros componentes de la muestra, que acompañan al agente psicoactivo. Estas impurezas pueden ser residuos de la síntesis química, de la extracción o la purificación. El perfil de impurezas es la información básica para los estudios de origen y de redes de distribución.

En su revisión del tema de la derivación de sustancias psicoactivas, Lin et al. ${ }^{[1]}$ mencionan 9 razones para utilizar la derivación química. Puede ser la búsqueda de formas químicas más compatibles con el ambiente cromatográfico, el favorecimiento de algún mecanismo de separación, el mejoramiento de la resolución, o de la elucidación estructural. Sin embargo, se ha identificado a la etapa de obtención de derivados como la principal fuente de prolongación y demoras en la ejecución de la marcha analítica. Una línea de desarrollo importante ha sido la búsqueda de esquemas de derivación cuantitativos, pero de duración corta y de aplicación fácil. 


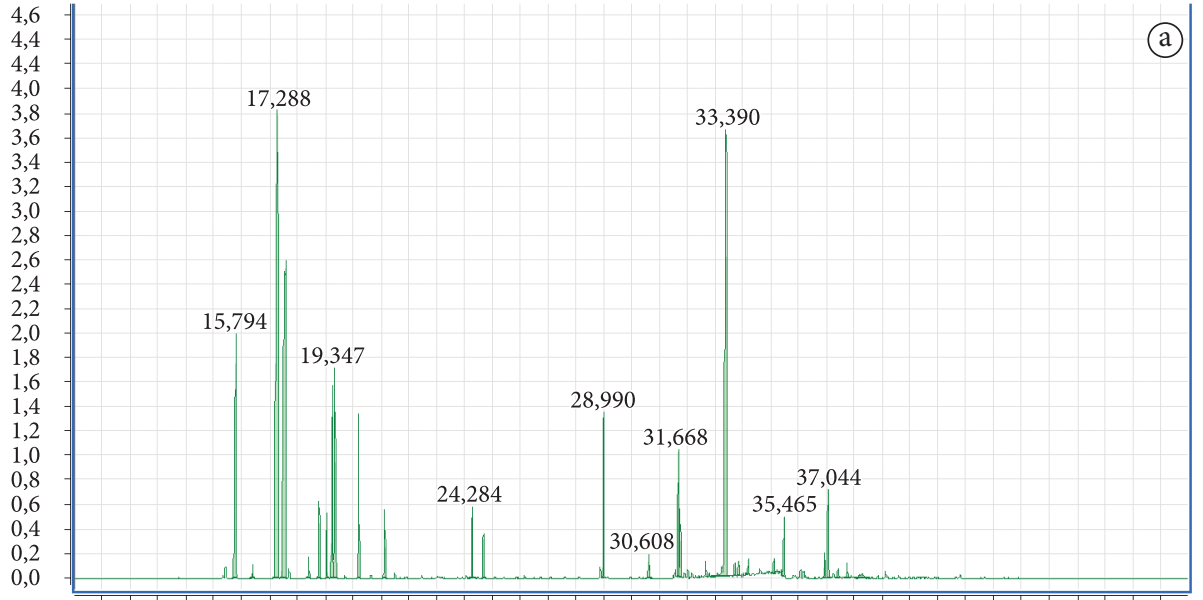

111213141516171819202122232425262728293031323334353637383940414243444546474849

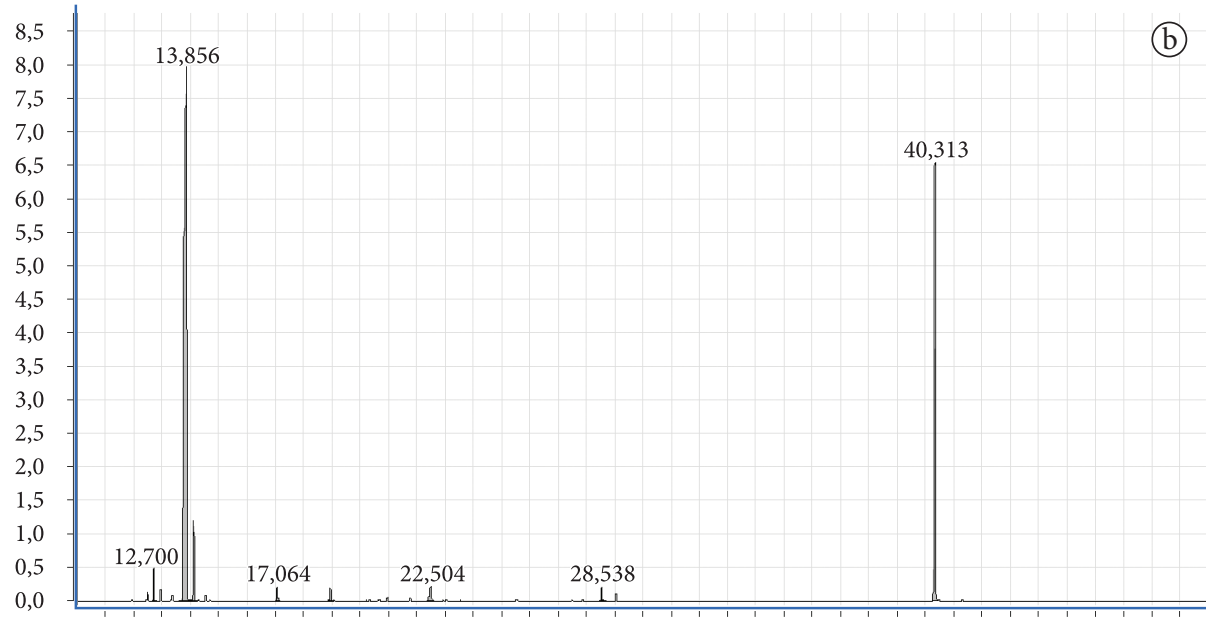

111213141516171819202122232425262728293031323334353637383940414243444546474849

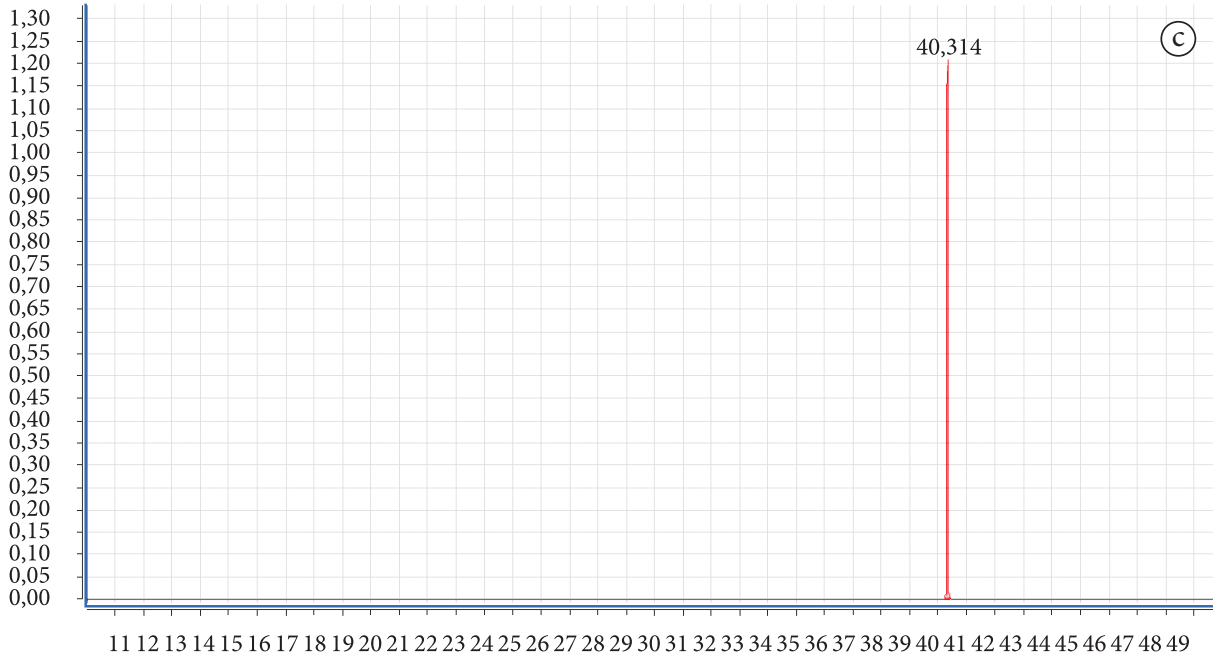

Figura 3 Extracto de una bebida (infusión) de varias plantas, que contiene trazas de cocaína. a) Corriente iónica total obtenida en modo full scan; b) Fragmentograma de masas (modo SIM), obtenido con base en los iones-diagnóstico de la cocaína, i.e., m/z 82, 182, 303; y c) Registro de la transición iónica 303 $\rightarrow 182$, usando la configuración tándem QqQ-MS. La máxima especificidad en la detección de la cocaína se logra usando el registro de esta transición de iones, precursor y producto, característica de la cocaína, 303 $\rightarrow 182$. 
Un primer ejemplo de los avances recientes es el análisis GC-MS aplicado a la determinación de anfetaminas en orina. A nivel mundial, el uso de estimulantes ha crecido bastante en la última década. Dentro de este grupo se destacan la anfetamina (AMP, "speed"), la metanfetamina (MET, “ice”) y la metilendioximetanfetamina (MDMA, "éxtasis"), en los primeros lugares. Tienen amplio uso las llamadas "drogas de clubes", que además de la MDMA, incluyen a la metilendioxianfetamina (MDA, "Adán”), metilendioxietilanfetamina (MDEA, "Eva") y la N-metil-1-(3,4 metilendioxifenil)-2-butanamina (MBDB). Marais y Laurens. ${ }^{[2]}$ desarrollaron un método analítico para el análisis rápido de orina en el cual se aprovecha la técnica de derivación extractiva para realizar en un solo paso la derivación de aminas con anhídrido heptafluorobutírico. La combinación de este método de derivación con el uso de la cromatografía rápida (columna DB-5 de $5 \mathrm{~m} \times 100 \mu \mathrm{m}, \mathrm{d}_{\mathrm{f}} 0,1 \mu \mathrm{m}$ ) permitió lograr un tiempo total de análisis de solamente 6 minutos por muestra. Gracias a la adquisición de datos en modo SIM, el método confirmatorio permitió alcanzar niveles de detección entre 1,5 y $6,2 \mathrm{ng} \mathrm{mL}^{-1}$ en la determinación simultánea de AMP, MET, metcatinona, efedrina, norefedrina, MDA, MDEA y MBDB.

Merece destacarse como el aprovechamiento efectivo de la derivación para el análisis de drogas, el método validado en el Instituto Nacional de Salud y Bienestar de Finlandia ${ }^{[3]}$, para la determinación de 50 drogas de abuso diferentes, en una muestra de $1 \mathrm{~mL}$ de saliva. La muestra se somete a extracción con acetato de butilo y luego de centrifugación, el sobrenadante se divide en 2 porciones y la fase acuosa constituye la tercera fracción. La primera porción orgánica se deriva con MSTFA (N-metil-N-(trimetilsilil) trifluoroacetamida) para su análisis por GC-MS con ionización por impacto de electrones. Los analitos a determinar en esta fracción son todas las drogas diferentes de las benzodiacepinas y la benzoílecgonina. Para la detección y la cuantificación se utilizaron sustancias patrón y el modo de adquisición SIM, para el cual, además de grupos particulares con valores $\mathrm{m} / \mathrm{z}$ típicos de los analitos de interés, se estudiaron las ventanas temporales apropiadas para su aplicación, con base en los tiempos de retención. La fracción 2 se pasa por una columna de extracción en fase sólida, que se eluye con una mezcla de alcohol isopropílico y acetato de etilo y se deriva con MTBSTFA [(N-metil-N-(tert-butildimetilsilil) trifluoroacetamida)]. La solución resultante se analiza por GC-MS con ionización química de iones negativos y tal como en el caso anterior, la adquisición se realiza en modo SIM, con grupos de iones $y$ ventanas de $t_{R}$ ajustadas para lograr la mejor cuantificación de benzodiacepinas patrón. Para la determinación de benzoilecgonina, la fase acuosa de la primera extracción con acetato de butilo se extrae con diclorometano. La fase orgánica se deriva con MSTFA y se analiza por GC-MS en modo SIM. Este protocolo analítico se le aplicó a 4183 muestras de saliva tomadas a choferes en Finlandia que quisieron participar voluntariamente. La sustancia controlada que apareció con mayor frecuencia en las muestras $(7,2 \%)$, que tuvieron algún resultado positivo para los 50 analitos de interés, fue el citalopram ( $1,5 \%$ de las muestras), un medicamento antidepresivo. Una de las ventajas del método desarrollado es que utilice una muestra de fácil consecución tal como el fluido oral. Hay un interés creciente en su uso como matriz de muestra alternativa a la sangre, que es la que se usa convencionalmente. El volumen de muestra de fluido oral es menor y las concentraciones de algunas drogas pueden ser mucho menores que en la sangre. Esto demanda mayor sensibilidad y selectividad del método analítico, que debe 
detectar y cuantificar varios analitos presentes en baja concentración en un volumen pequeño de muestra. Gracias a los avances instrumentales de la última década, es posible satisfacer los requerimientos que impone el análisis de fluido oral.

En comparación con la orina y la sangre, el fluido oral pertenece al grupo de especímenes denominados no-convencionales. En este conjunto se incluyen cabello, sudor, meconio, uñas y lágrimas. Presentan como ventaja sustancial que su recolección no es invasiva y puede realizarse bajo supervisión, lo cual es importante para muchas situaciones forenses y para el control de doping en los deportes. Existen revisiones sobre el análisis de drogas en cabello ${ }^{[4,5]}$, en saliva/fluido

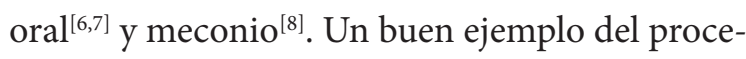
dimiento utilizado en el análisis de muestras de cabello es el método desarrollado por Cordero y Peterson ${ }^{[9]}$, que permite determinar en un solo cabello 18 analitos diferentes, entre opioides, anfetaminas, cocaína, diazepan y su metabolito. Una muestra de 10 a $50 \mathrm{mg}$ de cabello se extrae con $\mathrm{HCl} 0,1 \mathrm{~N}$ a $50{ }^{\circ} \mathrm{C}$, por 16 horas. El extracto neutralizado se pasa por una columna de extracción en fase sólida, que se eluye con cloroformo-isopropanol (drogas neutras) seguido por cloroformo-isopropanol-hidróxido de amonio (drogas básicas), para luego obtener los correspondientes derivados trimetilsilílicos. El análisis GC-MS se realiza en modo SIM, empleando para cada analito 3 iones diagnóstico y ventanas de tiempo establecidas con los tiempos de retención obtenidos según el perfil cromatográfico adquirido en modo de barrido completo.

A pesar de la relativa facilidad de muestreo, los especímenes alternativos todavía no se usan comúnmente. En su revisión sobre el uso de meconio, uñas y lágrimas en el análisis de drogas, Katarzyn Madej ${ }^{[10]}$ considera que una de las razones principales para el uso poco amplio de estas muestras es que los métodos analíticos requieren instrumentación costosa, de altas selectividad y sensibilidad (LC-MS, LC-MS²). Cuando la técnica analítica es GC-MS, la determinación de sustancias controladas requiere en la mayoría de los casos la inclusión de un paso de derivación, lo cual se suma a una metodología extensa de preparación, en los casos de uñas, cabello o meconio. Aunque el análisis de meconio implica un procedimiento relativamente complicado para la eliminación de interferencias, suministra información valiosa sobre el consumo posible de drogas durante los últimos 6 meses de la gestación. Otra fuente de información complementaria es el análisis de leche materna. Fríguls et al. ${ }^{[11]}$ han hecho una revisión sobre este tema, en la que presentan para diferentes tipos de drogas, tanto lícitas como ilícitas, su farmacocinética, efectos adversos sobre los niños y los procedimientos publicados sobre su determinación en leche materna. Los métodos de preparación de muestra generalmente incluyen un paso de extracción líquido-líquido o de extracción en fase sólida. Tal como ya se ha mencionado, para su determinación por GC-MS, muchas drogas requieren una derivación química que aumente su volatilidad.

Todos los tipos de muestra mencionados se emplean para establecer el posible consumo de drogas por parte de individuos. En los años recientes se han realizado estudios sobre el consumo de drogas lícitas e ilícitas por parte de poblaciones completas. Para esto, los objetos de estudio han sido las aguas residuales municipales. En el método propuesto por el Instituto de Análisis e Investigación de Alimentos de Santiago de Compostela ${ }^{[12]}$, se realiza primero una extracción en fase sólida. Por reacción con MSTFA, del extracto se obtienen derivados trimetilsilílicos, que se separan por cromatografía de gases y se identifican y cuantifican en un analizador de masas de trampa iónica. Para lograr selectividad, inicialmente se realizan barridos 
en el intervalo $m / z$ 70-500 y luego, la trampa de iones se opera en modo resonante MS/MS para los valores de los iones padre y producto correspondientes a 18 analitos de interés y sus análogos deuterados. Dentro de los analitos de interés se incluyeron cocaína, benzoílecgonina, codeína, morfina, tetrahidrocanabinol, MDMA, MDA y MDEA. Aplicado a muestras reales, el método confirmó el ingreso de estos analitos al ambiente a través de las aguas residuales municipales.

\section{La cromatografía de gases multidimensional y la perfilación}

La sustancia que llega al consumidor de drogas ilícitas es una mezcla de agentes activos con muchos otros componentes que surgen de los procesos de extracción o síntesis química, de purificación y de dilución. La identificación de estos otros componentes y la determinación de sus cantidades relativas, permiten construir un perfil químico o perfil de impurezas, que distingue al producto y sirve de base para investigar los orígenes y las redes de distribución de las drogas ilícitas. Por ejemplo, la metanfetamina se puede sintetizar por los procedimientos sintéticos de Emde o de Nagai ${ }^{[13,14]}$. En el primer caso quedan residuos de cloroefedrina y en el segundo caso, las impurezas comunes son 1,3-dimetil-2-fenilnaftaleno y 1-bencil-3-metilnaftaleno. El análisis GC-MS de metanfetaminas decomisadas en Korea permitió establecer que el $80 \%$ de las muestras provino del método sintético de Emde y que en los 2 años previos aumentó en 30\% el número de muestras sintetizadas por el método de Nagai ${ }^{[15]}$.

En los últimos años, se han hecho esfuerzos llamados de "armonización", para establecer criterios de calidad en el análisis químico para la perfilación de drogas, que permitan la comparación de resultados de laboratorios de diferentes países e instituciones $^{[16-18]}$. El reto analítico lo constituye la gran diversidad de estructuras químicas posibles dentro del conjunto de impurezas. En los métodos presentados en las secciones anteriores de este artículo hay protocolos diseñados para cuantificar drogas ilícitas individuales, mientras que otros se pueden aplicar a la determinación simultánea de mezclas multicomponente de drogas y sus metabolitos. Aunque se pueden manejar conjuntos del orden de 50 analitos diferentes, se dispone de sustancias patrón para cada uno y con éstos se calibra y optimiza el desempeño del método analítico. En todos estos casos se utiliza la cromatografía unidimensional y la detección y cuantificación de alta selectividad se buscan con la adquisición de corrientes iónicas en modo SIM. Para el propósito de obtener el perfil químico, el gran número de analitos requiere que se aumente la resolución cromatográfica. Por esto, si bien los métodos de análisis GC-MS de drogas ilícitas se usan de manera rutinaria en tareas de cuantificación para analitos específicos, las técnicas multidimensionales han encontrado gran aplicación en el campo de la perfilación de drogas y se han extendido a otros estudios forenses relacionados.

Kolbrich et al. ${ }^{[19]}$ utilizaron una técnica 2D-GC/MS para el análisis de plasma humano, en la que una válvula de conmutación de tecnología microfluídica (Deans switch) traslada secciones del flujo de una columna cromatográfica a otra de diferente fase estacionaria, a través de una trampa criogénica de calentamiento programable. El uso de adquisición en modo SIM permitió lograr límites de cuantificación entre 1,0 y $2,5 \mu \mathrm{g} \mathrm{L} \mathrm{L}^{-1}$ para MDA, MDEA, MDMA, 4-hidroxi-3-metoximetanfetamina (HMMA) y 4-hidroxi-3-metoxianfetamina (HMA). Análogos perdeuterados de estas sustancias sirvieron como patrones internos. El uso de la cromatografía bidimensional permi- 
tió el desarrollo de este método para el análisis simultáneo de 5 aminas relacionadas estructuralmente, cuyos tiempos de retención son cercanos.

Se han desarrollado métodos basados en 2D-GC/MS para diversas drogas ilícitas. Un ejemplo de los avances logrados lo constituye el análisis de marihuana, el cual demanda alta resolución porque aunque el tetrahidrocanabinol es la principal sustancia activa, puede estar junto con más de 60 canabinoides diferentes, que se han detectado en Cannabis sativa. Se han reportado métodos 2D-GC/MS para determinar estos analitos en sangre ${ }^{[20]}$, plasma humano ${ }^{[21,22]}$, fluido oral ${ }^{[23,24]}$, cabello $^{[25]}$ y meconio ${ }^{[26]}$. Estos son métodos exitosos, que logran una buena separación con alta sensibilidad. Sin embargo, mezclas más complejas requieren un número alto de cortes (heart-cuttings) que se redirigen hacia la segunda columna y no siempre se logra trasladar todos los analitos de interés. Para atender estas situaciones y en general todos aquellos casos en donde se tienen mezclas complejas con constituyentes de naturaleza desconocida, se recurre al siguiente nivel de desarrollo, que es la cromatografía completa, GC $\times$ GC. En esta técnica, todo el efluente de la primera columna se traslada a la segunda columna por medio de un sistema periódico de conmutación con crioenfocamiento $^{[27]}$. De esta forma, todos los compuestos coeluidos en la primera columna pueden, en principio, separarse en la segunda columna, que tiene una fase estacionaria con polaridad opuesta (ortogonal) a la de la primera columna. En comparación con la cromatografía unidimensional, GC $\times$ GC tiene una capacidad mucho mayor de resolución de componentes y proporciona una relación $\mathrm{S} / \mathrm{N}$ más alta. A estas ventajas se suma otra de utilidad especial en el caso de la perfilación de drogas, como es la formación de grupos de sustancias de naturaleza química similar, en el mapa bidimensional que se obtiene como respuesta de la técnica GC $\times$ GC. Esto facilita la comparación de muestras y permite establecer criterios de similitud. En un estudio de 24 tabletas de éxtasis, la comparación de los perfiles bidimensionales mostró la existencia de 8 grupos, según su contenido de impurezas. El análisis de componentes principales aplicado al conjunto de datos reveló claramente la formación de grupos según el país de origen ${ }^{[28]}$.

En su revisión de la aplicación de la cromatografía completa al análisis de drogas ilícitas, Mitrevski, Wynne y Marriott. ${ }^{[29]}$ destacaron que el espectrómetro de masas que mejor satisface la velocidad de barrido que requiere la técnica $\mathrm{GC} \times \mathrm{GC}$ es el de analizador de tiempo de vuelo (TOF). El principal obstáculo a vencer para aumentar la aplicación de la cromatografía completa es el manejo de los datos. Se requieren herramientas que faciliten el manejo del gran volumen de información que genera la técnica, para que el usuario pueda realizar de manera sencilla tareas como deconvolución, búsqueda de agrupaciones según diversos criterios y la aplicación de técnicas de la estadística multivariable.

Aunque en GC $\times$ GC los mejores resultados se obtienen con un analizador de tiempo de vuelo, ya existen algunas aplicaciones en las que se usa un analizador cuadrupolar. Song et al. ${ }^{[30]}$ reportaron la detección de 77 componentes en una mezcla de drogas por medio de GC $\times$ GC con analizador cuadrupolar, cuyo rango de masas no cubría todo el espectro de masas sino un intervalo pequeño, que podría corresponder a los extremos inferior o superior del espectro para, de esta forma, lograr tiempos de barrido cortos, compatibles con las necesidades de la técnica de cromatografía rápida (segunda columna).

El carácter no específico, de amplio espectro, del análisis GC $\times$ GC, se ilustra en el trabajo publicado por Guthery et al. ${ }^{[31]}$, sobre análisis de drogas en muestras de cabello por técnicas 
LC-MS y GC $\times$ GC-TOF/MS. Mientras que los métodos LC-MS permitieron la cuantificación de codeína, morfina, anfetamina, metanfetamina, metadona, bencilpiperazina y MDMA, el análisis GC × GC-TOF/MS logró detectar la presencia no prevista de cocaína, diazepan y metacualona. La identificación se logró por comparación de los espectros de masas con los de bases de datos. Tal como en las metodologías GC-MS unidimensionales, la preparación de la muestra incluye una etapa de derivación, generalmente, hacia los derivados trimetilsilílicos (con MSTFA), para mejorar las propiedades cromatográficas de los analitos que posean grupos funcionales con hidrógenos lábiles. Aunque los métodos para analitos específicos suministran sensibilidades altas, su uso en tareas de tamizaje para un número grande de compuestos está obstaculizado por los altos costos y el mayor tiempo que implica realizar varios análisis por muestra. Para estos casos, GC $\times$ GC TOF/MS tiene la gran ventaja de que se realiza un solo análisis por muestra, con alta sensibilidad. Además, la información espectral permite la identificación de sustancias o contaminantes desconocidos, lo cual no se logra con los métodos para analitos específicos.

Además de la resolución cromatográfica, en las técnicas acopladas GC-MS la espectrometría de masas aporta la posibilidad de alcanzar altas sensibilidades y selectividades. En el caso de la espectrometría de masas de alta resolución, la gran capacidad de discriminación que se obtiene en la detección de masas exactas permite lograr en acoplamiento con la cromatografía unidimensional, resultados equivalentes o superiores a los que se obtienen con GC $\times$ GC-TOF/ MS cuando en esta última técnica la resolución másica es unitaria. Un ejemplo del uso de la espectrometría de masas de alta resolución para la obtención de alta sensibilidad es el método desarrollado por Georgakopoulos et al. ${ }^{[32]}$ para la determinación de esteroides en orina, que utiliza tanto LC-TOF/MS como GC-TOF/MS. Luego de la extracción líquido-líquido de la muestra de orina hidrolizada, se obtienen los derivados trimetilsilílicos para su análisis GC-TOF/MS y una alícuota sin derivar se analiza por LC-TOF/MS. El método permitió alcanzar niveles de detección de entre 10 y $500 \mathrm{ng} \mathrm{mL}^{-1}$ para agentes anabólicos, corticosteroides, estimulantes, bloqueadores $\beta$, diuréticos y narcóticos. El uso de la ionización por impacto de electrones en sistemas GC-MS de alta resolución presenta el inconveniente de que junto con la producción de una alta fragmentación molecular, genera dificultades para la identificación por comparación espectral con bases de datos, ya que todavía están en desarrollo las bases de datos espectrales de alta resolución. El uso de métodos de ionización suave en combinación con la alta resolución podría ser una buena alternativa. Sin embargo, se pueden obtener cromatogramas con menor ruido químico si se extraen de la corriente iónica total los valores de masas dentro de ventanas o intervalos de tiempo angostos. Bajo estas condiciones se mejora considerablemente la comparación con espectros obtenidos en analizadores convencionales ${ }^{[33]}$.

\section{Conclusiones}

La técnica GC-MS se utiliza exitosamente en el análisis de drogas de uso ilícito, tanto en la determinación de sustancias específicas como en la obtención de los perfiles de composición química, en aplicaciones desarrolladas para satisfacer diversos propósitos clínicos, toxicológicos o forenses. La gran diversidad de estructuras químicas tanto de las sustancias activas como de sus contaminantes, así como de las matrices en las que se realiza el examen, hacen que la preparación de la muestra juegue un papel determinante. 
Los avances tanto en la separación cromatográfica (GC $\times$ GC) como en la detección espectrométrica (SIM, MS/MS, alta resolución) permiten alcanzar sensibilidades ( 1 en $10^{9}$ ) y especificidades altas en el análisis de mezclas complejas que contienen drogas de uso ilícito.

\section{Referencias}

1 Dong-Liang L, Sheng-Meng W, Chih-Hung W, Bud-Gen C, Ray L. Chemical derivatization for the analysis of drugs by GC-MS - A conceptual review. Journal of Food and Drug Analysis 2008; 16(1):1-10.

2 Marais A, Laurens J. Rapid GC-MS confirmation of amphetamines in urine by extractive acylation. Forensic Science International 2009; 183:78-86. http:// dx.doi.org/10.1016/j.forsciint.2008.10.021

3 Langel K, Gunnar T, Ariniemi K, Rajamäki, O, Lillsunde P. A validated method for the detection and quantitation of 50 drugs of abuse and medicinal drugs in oral fluid by gas chromatographymass spectrometry. Journal of Chromatography B 2011; 879:859-870. http://dx.doi.org/10.1016/j. jchromb.2011.02.027

4 Sachs H, Kintz P. Testing for drugs in hair: Critical review of chromatographic procedures since 1992. Journal of Chromatography B 1998; 713:147-161. http://dx.doi.org/10.1016/S0378-4347(98)00168-6

5 Pragst F, Balikova M. State of the art in hair analysis for detection of drug and alcohol abuse. Clinica Chimica Acta 62006; 370:17-49. http://dx.doi.org/10.1016/j. cca.2006.02.019

6 Drummer O. Introduction and review of collection techniques and applications of drug testing of oral fluid. Therapeutic Drug Monitoring 2008; 30:203-206.

7 Schramm W, Smith R, Craig P, Kidwell D. Drugs of abuse in saliva: A review. Journal of Analytical Toxicology 1992; 16:1-9.

8 Moore C, Negrusz A, Lewis D. Determination of drugs of abuse in meconium. Journal of Chromatography B 1998; 713:137-146. http://dx.doi.org/10.1016/ S0378-4347(97)00479-9

9 Cordero R, Paterson S. Simultaneous quantification of opiates, amphetamines, cocaine and metabolites and diazepam and metabolite in a single hair sample using GC-MS. Journal of Chromatography B 2007; 850:423431. http://dx.doi.org/10.1016/j.jchromb.2006.12.021
10 Madej K. Analysis of meconium, nails and tears for determination of medicines and drugs of abuse. Trends in Analytical Chemistry 2010; 29(3):246-259. http://dx.doi.org/10.1016/j.trac.2010.01.005

11 Fríguls B, Joya X, García-Algar O, Pallás C, Vall O, Pichini S. A comprehensive review of assay methods to determine drugs in breast milk and the safety of breastfeeding when taking drugs. Analytical and Bioanalytical Chemistry 2010; 397:1157-1179. http:// dx.doi.org/10.1007/s00216-010-3681-0

12 González-Mariño I, Quintana J, Rodríguez I, Cela R. Determination of drugs of abuse in water by solid-phase extraction, derivatisation and gas chromatography-ion trap-tandem mass spectrometry. Journal of Chromatography A 2010; 1217:1748-1760. http://dx.doi.org/10.1016/j.chroma.2010.01.046

13 Kishi $\mathrm{T}$, Inoue $\mathrm{T}$, Suzuke $\mathrm{S}$, Yasuda $\mathrm{T}$, Okikawa T, Niwaguchi T. Analysis of impurities in methamphetamine. Eisei Kagaku 1983; 29:39- 48. http://dx.doi.org/10.1248/jhs1956.29.6_400

14 Emde H. Über diastereomerie I. Konfiguration des ephedrines. Helvetica Chimica Acta 1929; 12:365-376. http://dx.doi.org/10.1002/hlca.19290120142

15 Ko B, Suh S, Suh Y, In M, Kim S. The impurity characteristics of methamphetamine synthesized by Emde and Nagai method. Forensic Science International 2007;170:142-147. http://dx.doi. org/10.1016/j.forsciint.2007.03.029

16 Strömberg L, Lundberg L, Neumann H, Bobon B, Huizer H, Van Der Stelt N. Heroin impurity profiling. A harmonization study for retrospective comparisons. Forensic Science International 2000; 114:67-88. http:// dx.doi.org/10.1016/S0379-0738(00)00295-4

17 Lociciro S, Hayoz P, Esseiva P, Dujourdy L, Besacier F, Margot P. Cocaine profiling for strategic intelligence purposes, a cross-border project between France and Switzerland. Part I. Optimisation and harmonisation of the profiling method. Forensic Science International 2007; 167:220-228. http://dx.doi. org/10.1016/j.forsciint.2006.06.052

18 Weyermann C, Marquis R, Delaporte C, Esseiva P, Lock E, Aalberg L et al. Drug intelligence based on MDMA tablets data I. Organic impurities profiling. Forensic Science International 2008; 177:11-16. http:// dx.doi.org/10.1016/j.forsciint.2007.10.001

19 Kolbrich E, Lowe R, Huestis M. Two-dimensional gas chromatography/electron-impact mass spectrometry with cryofocusing for simultaneous quantification of 
MDMA, MDA, HMMA, HMA, and MDEA in human plasma. Clinical Chemistry 2008; 54(2):379-387. http://dx.doi.org/10.1373/clinchem.2007.096800

20 Scurlock R, Ohlson G, Worthen D. The detection of $\Delta^{9}$-tetrahydrocannabinol (THC) and 11-nor-9carboxy- $\Delta^{9}$-tetrahydrocannabinol (THCA) in whole blood using two-dimensional gas chromatography and EI-mass spectrometry. Journal of Analytical Toxicology 2006; 30:262-266.

21 Lowe R, Karschner E, Schwilke E, Barnes A, Huestis M. Simultaneousquantification of $\Delta^{9}$-tetrahydrocannabinol, 11-hydroxy- $\Delta^{9}$-tetrahydrocannabinol, and 11-nor- $\Delta^{9}$ tetrahydrocannabinol-9-carboxylic acid in human plasma using two-dimensional gas chromatography, cryofocusing, and electron impact-mass spectrometry. Journal of Chromatography A 2007; 1163:318-327. http://dx.doi.org/10.1007/s00216-010-3599-6

22 Karschner E, Barnes A, Lowe R, Scheidweiler K, Huestis M. Validation of a two-dimensional gas chromatography mass spectrometry method for the simultaneous quantification of cannabidiol, $\Delta$ 9-tetrahydrocannabinol (THC),11-hydroxy-THC, and 11-nor-9-carboxy-THC in plasma. Analytical and Bioanalytical Chemistry 2010; 397:603-611. http:// dx.doi.org/10.1007/s00216-010-3599-6

23 Moore C, Coulter C, Rana S, Vincent M, Soares J. Analytical procedure for the determination of the marijuana metabolite 11-nor- $\Delta^{9}$-tetrahydrocannabinol9-carboxylic acid in oral fluid specimens. Journal of Analytical Toxicology 2006; 30:409-412.

Moore C, Rana S, Coulter C, Day D, Vincent $\mathrm{M}$, Soares J. Detection of conjugated 11-nor- $\Delta^{9}-$ tetrahydrocannabinol-9-carboxylic acid in oral fluid. Journal of Analytical Toxicology 2007; 31:187-194.

25 Moore C, Rana S, Coulter C, Feyerherm F, Prest H. Application of two-dimensional gas chromatography with electron capture chemical ionization mass spectrometry to the detection of 11-nor- $\Delta^{9}$ tetrahydrocannabinol-9-carboxylic acid (THC-COOH) in hair. Journal of Analytical Toxicology 2006; 30:171-177.

26 Marin S, Coles R, Urry F, McMillin G. Confirmation of cannabinoids in meconium using two-dimensional gas chromatography with mass spectrometry detection.
Journal of Chromatography B 2007; 858:59-64. http:// dx.doi.org/10.1016/j.jchromb.2007.08.026

27 Harynuk J, Marriott P. GS-MS principles and instrumentation: comprehensive two-dimensional gas chromotography mass spectrometry. In: Caprioli R, editor. The Encyclopedia of Mass Spectrometry. Oxford: Elsevier; 2006. vol. 8: Hyphenated Methods, cap. 2, p. 83-92.

28 Mitrevski B, Veleska B, Engel E, Wynne P, Song S, Marriott P. Chemical signature of ecstasy volatiles by comprehensive two-dimensional gas chromatography. Forensic Science International 2011; 209:11-20. http://dx.doi. org/10.1016/j.forsciint.2010.11.008

29 Mitrevski B, Wynne P, Marriott P. Comprehensive two-dimensional gas chromatography applied to illicit drug analysis. Analytical and Bioanalytical Chemistry 2011; 401:2361-2371. http://dx.doi. org/10.1007/s00216-011-5234-6

30 Song S, Marriott P, Wynne P. Comprehensive two-dimensional gas chromatography with timeof-flight mass spectrometry (GC $\times$ GC-TOFMS) for drug screening and confirmation. Journal of Chromatography A 2004; 1058:223-232. http://dx.doi. org/10.1016/S0021-9673(04)01444-X

31 Guthery B, Bassindale T, Bassindale A, Pillinger C, Morgan G. Qualitative drug analysis of hair extracts by comprehensive two-dimensional gas chromatography/time-of-flight mass spectrometry. Journal of Chromatography A 2010; 1217:4402-4410. http://dx.doi.org/10.1016/j.chroma.2010.04.020

32 Georgakopoulos C, Vonaparti A, Stamou M, Kiousi P, Lyris E, Angelis Y et al. Preventive doping control analysis: liquid and gas chromatography time-of-flight mass spectrometry for detection of designer steroids. Rapid Communications in Mass Spectrometry 2007; 21:2439-2446. http://dx.doi. org/10.1002/rcm.3103

33 Pavlic M, Libiseller K, Oberacher H. Combined use of ESI-QqTOF-MS and ESI-QqTOF-MS/ MSwith mass-spectral library search for qualitative analysis of drugs. Analytical and Bioanalytical Chemistry 2006; 386:69-82. http://dx.doi. org/10.1007/s00216-006-0634-8 